\title{
Tensor Based Tumor Tissue Type Differentiation Using Magnetic Resonance Spectroscopic Imaging
}

\author{
H. N. Bharath ${ }^{1,2}$, D. M. Sima ${ }^{1,2}$, N. Sauwen ${ }^{1,2}$, U. Himmelreich ${ }^{3}$, L. De Lathauwer ${ }^{1,4}$, S. Van Huffel ${ }^{1,2}$
}

\begin{abstract}
Magnetic resonance spectroscopic imaging (MRSI) has the potential to characterise different tissue types in brain tumors. Blind source separation techniques are used to extract the specific tissue profiles and their corresponding distribution from the MRSI data. A 3-dimensional MRSI tensor is constructed from in vivo 2D-MRSI data of individual tumor patients. Non-negative canonical polyadic decomposition (NCPD) with common factor in mode-1 and mode-2 and $l_{1}$ regularization on mode-3 is applied on the MRSI tensor to differentiate various tissue types. Initial in vivo study shows that NCPD has better performance in identifying tumor and necrotic tissue type in high grade glioma patients compared to previous matrix-based decompositions, such as non-negative matrix factorization and hierarchical non-negative matrix factorization.
\end{abstract}

\section{INTRODUCTION}

Accurate characterisation and localization of pathologic tissue types play a key role in diagnosis and treatment planning of brain tumors. The tumor region of glioblastoma multiforme (GBM) could consist of several tissue types, which represent actively growing tumor, necrosis or normal brain tissue [1]. In recent years, many MR modalities are being used for tumor diagnosis [2]. Magnetic resonance spectroscopic imaging (MRSI) is a noninvasive imaging technique which provides spectral profiles in a two or three dimensional voxel grid, from which the spatial distribution of metabolite concentrations can be estimated. MRSI has been successfully applied to diagnosis and prognosis of brain tumors. There are many algorithms for MRSI data analysis available in the literature that aim at tissue characterisation, tumor localization and classification. An independent component analysis based method was presented in [3], while canonical correlation analysis was used for tissue segmentation in [4]. Hierarchical non-negative matrix factorization (hNMF) has been applied to brain MRSI data to differentiate different tissue patterns in GBM patients [1]. The performance of hNMF [1] deteriorates in the presence of artifacts because it can handle only three tissue types.

In this paper, a tensor based algorithm is proposed for tissue type differentiation in tumor patients from 2D-MRSI

*This research was supported by ERC Advanced Grant, \#339804 BIOTENSORS. This paper reflects only the authors views and the Union is not liable for any use that may be made of the contained information.

${ }^{1}$ Department of Electrical Engineering (ESAT), STADIUS Center for Dynamical Systems, Signal Processing and Data Analytics, KU Leuven, Leuven, Belgium.

${ }^{2}$ iMinds Medical Information Technologies, Leuven, Belgium.

${ }^{3}$ Biomedical MRI Unit/Molecular Small Animal Imaging Center, Department of Imaging and Pathology, KU Leuven, Leuven, Belgium.

${ }^{4}$ The Group of Science, Engineering and Technology, KU Leuven Kulak, Kortrijk, Belgium. signals. The proposed method consists of representing the 2D-MRSI signals in a 3-dimensional tensor and applying non-negative canonical polyadic decomposition (NCPD) with $l_{1}$ regularization to the MRSI tensor. Preliminary results of the proposed algorithm on the in vivo study are presented in section IV. Performance of the NCPD algorithm was evaluated using expert labeling and compared with singlestage NMF and hNMF.

\section{MATERIALS}

\section{A. Data acquisition protocol}

Short-TE MRSI data from nine patients were acquired on a 3T MR scanner (Achieva, Philips, Best, The Netherlands) at the University Hospital of Leuven. Point-resolved spectroscopy (PRESS) was used as the volume selection technique, TR/TE: $2000 / 35 \mathrm{~ms}$, FOV: $16 \mathrm{~cm} * 16 \mathrm{~cm}$, volume of interest (VOI): $8 \mathrm{~cm} * 8 \mathrm{~cm}$, acquisition voxel size: $1 \mathrm{~cm} * 1 \mathrm{~cm}$, slice thickness: $1 \mathrm{~cm}$. The study and the experimental procedures involving human subjects have been approved by the ethical committee of the institute.

\section{B. Data processing}

The residual water component was removed from the MRSI data using Hankel Lanczos singular value decomposition with partial reorthogonalization (HLSVD-PRO) [5]. A model order of 30 and a passband of 0.25 to 4.2 parts per million (ppm) was used in HLSVD-PRO algorithm. After removing the water component baseline correction and baseline offset correction was performed. Pre-processing was done using the Matlab based in-house software, SPID [6].

The spectra were aligned in frequency using a simulated reference spectrum, where the reference spectrum was generated using the parameters given in [7]. The pre-processed spectra were truncated to the region $0.25-4.2 \mathrm{ppm}$ and the truncated spectra were normalized to unit norm $\left(l_{2}\right)$. Voxels outside the MRSI PRESS excitation volume are excluded from the analysis.

\section{Tensor COnstruction And Non-Negative CPD}

\section{A. MRSI tensor construction}

For each voxel in the MRSI grid, a reduced spectrum $X$ is constructed from the corresponding pre-processed spectrum. Elements of the vector $X$ are obtained by moving an overlapping window over the spectrum, where the $i^{\text {th }}$ element of $X$ is the sum of squares of all the elements in the $i^{\text {th }}$ window segment,

$$
X(i)=\sum_{j=1}^{L} s_{i j}^{2}
$$


$s_{i}$ is the spectrum at the $i^{\text {th }}$ segment and $L$ is the length of the window segment. Fig. 1a shows the construction of vector $X$ from the spectrum. The resulting vector $X$ can be considered as a denoised and reduced-length version of the original spectrum. The window length $L$ is chosen such that it covers the widest peak in the spectra. Use of vector $X$ has two advantages:

1) It reduces the length of spectra without losing vital information required for tumor tissue type differentiation.

2) It gives more weight to the peaks and makes the signal smoother.

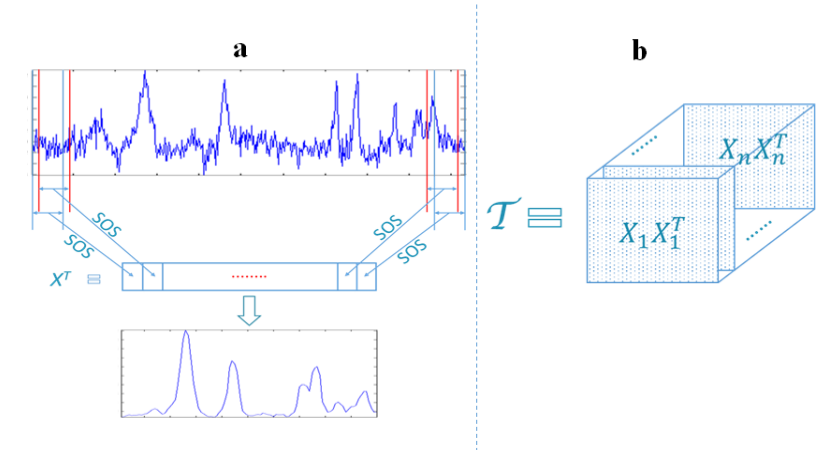

Fig. 1: (a) Construction of reduced spectrum, $X$ from the preprocessed spectra. SOS: sum of squares. (b) Construction of the MRSI tensor $\mathcal{T}$ from the reduced spectra, $X . n$ is the number of voxels.

A 3-way MRSI tensor $\mathcal{T}$ is constructed by stacking $X X^{T}$ from all the voxels in the MRSI grid as shown in Fig. 1(b).

\section{B. Non-Negative CPD}

Non-negative canonical polyadic decomposition (NCPD) is a tensor decomposition method, where the tensor is decomposed into a sum of rank-one tensors with non-negativity constraints on the factor matrices [8]. For a third-order tensor $x \in \mathbb{R}^{I \times J \times K}$, NCPD can be written as

$$
\chi \approx \llbracket A B C \rrbracket \equiv \sum_{r=1}^{R} a_{r} \circ b_{r} \circ c_{r}, \quad A, B, C \geq 0
$$

where $A=\left[a_{1}, a_{2}, \ldots, a_{R}\right] \in \mathbb{R}_{+}^{I \times R}, B=\left[b_{1}, b_{2}, \ldots, b_{R}\right] \in \mathbb{R}_{+}^{J \times R}$ and $C=\left[c_{1}, c_{2}, \ldots, c_{R}\right] \in \mathbb{R}_{+}^{K \times R}$ are non-negative factor matrices. $R$ is the rank, defined as the number of rank-one terms, ' $\circ$ ' represents outer product.

In the MRSI tensor $\mathcal{T}$, the frontal slices are symmetrical, therefore we constrain the frontal slices of each NCPD rankone term to be symmetric. To maintain symmetry, a common factor matrix is used for mode- 1 and mode- 2 in the NCPD as shown in Fig 2. After performing the NCPD on the MRSI tensor $\mathcal{T}$ we obtain two factor matrices $S$ and $H$, where $S$ represents the tissue specific patterns of the reduced spectra and $H$ represents the spatial distribution of each tissue type.

Each rank-one term obtained from the NCPD of the MRSI tensor $\mathcal{T}$ corresponds to a particular tissue type. Here, we assume that spectra corresponding to each voxel belong to a

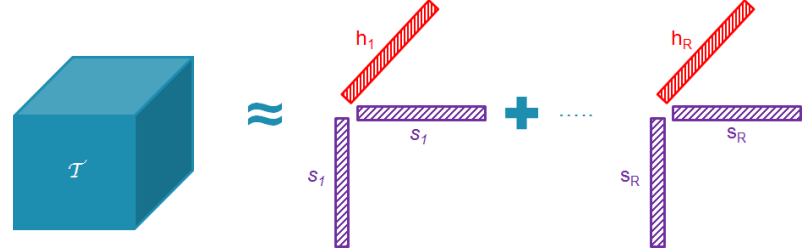

Fig. 2: Non-negative CPD of MRSI tensor $\mathcal{T}$ : MRSI tensor $\mathcal{T}$ is decomposed into $R$ rank-1 tensors. Common factor $S$ is used in mode- 1 and mode- 2 to maintain symmetry of frontal slices. Each $s_{i}$ gives a tissue specific reduced spectral pattern and the corresponding $h_{i}$ gives the spatial distribution of the respective tissue type. Non-negativity of $S$ and $H$ is imposed in the decomposition.

particular tissue type, therefore the factor matrix $H$ will be sparse, meaning that each row has only one high value. The sparsity in the factor matrix $H$ is achieved by imposing a $l_{1}$ regularization on $H$. The NCPD with $l_{1}$ regularization can be written as

$$
\begin{array}{r}
{\left[S^{*}, H^{*}\right]=\min _{S \geq 0, H \geq 0}\left\|\mathcal{T}-\sum_{i=1}^{R} S(:, i) \circ S(:, i) \circ H(:, i)\right\|_{2}^{2}} \\
+\lambda\|\operatorname{Vec}(H)\|_{1}
\end{array}
$$

where $S$ and $H$ are the aforementioned factor matrices and $\lambda$ is the parameter which controls the sparsity. In this work, the tensor decomposition was performed using the Tensorlab Matlab package [9]. Non-negativity constraints and $l_{1}$ regularization are applied using the structured data fusion method [10] available in Tensorlab.

\section{Recovering spectra from NCPD}

The NCPD of the MRSI tensor $\mathcal{T}$ gives the factor matrix $S$, which contains the reduced spectral patterns specific to different tissue types. However, it is also possible to recover tissue specific spectral sources as vectors of the same length as the pre-processed MRSI spectra, which are more interpretable since they can be directly compared to the original spectra. To this end, a least squares problem is solved, and the spectral sources representing the tissue specific spectral pattern $W$ are:

$$
W=\left(H^{\dagger} Y^{T}\right)^{T}
$$

where $H^{\dagger}$ is the Moore-Penrose pseudoinverse of the NCPD factor matrix $H$ and $Y$ is the matrix containing the unit normalized spectra from all voxels as its columns. The matrix $H$ is obtained from NCPD and it is based on the normalized spectra. The distribution of the sources in the original unnormalised spectra are more meaningful and represents true distributions. Therefore, spatial distribution $H_{D}$ of the different tissue types in the MRSI grid are obtained from unnormalised spectra and the spectral source $W$ using nonnegative least squares with $l_{1}$ regularization [11]. Tissue distribution maps are obtained by reshaping the rows of $H_{D}$ to the 2D-MRSI grid. 


\section{EXPERIMENTAL RESULTS}

The proposed NCPD algorithm is applied on in vivo ${ }^{1} \mathrm{H}$ MRSI data of high grade glioma patients. For each patient, all MRSI voxels have been labeled after visual inspection by an expert; the labeling consists of an assignment of each voxel to a tissue type (active tumor, necrosis, normal tissue, or a mixture). The performance evaluation of the algorithms on the in vivo study is analyzed using two measures. 1) Source correlation: the correlation between the estimated spectral sources and the tissue specific spectrum based on expert labeling of the in vivo MRSI voxels is calculated [1]. The tissue specific spectrum based on expert labeling for a particular tissue type is computed as the average of all the spectra from the voxels labelled by the expert as belonging to that tissue type. 2) Distribution correlation: the correlation between the estimated distribution map corresponding to a particular tissue type and the distribution map based on expert labeling is calculated. For each tissue type, a distribution map based on expert labeling is obtained by using values equal to the $l_{2}$ norm of the corresponding spectra for all voxels labeled as a certain tissue class, and values of 0 for the other voxels.

In order to evaluate performance, three algorithms, NCPD, single stage NMF and hNMF [1] were applied on 10 in vivo ${ }^{1} \mathrm{H}$ MRSI datasets from 9 patients with high grade glioma. For all the datasets, rank of $R=6$ is used in NCPD and NMF methods. Rank $R=6$ is chosen based on the prior knowledge and visual inspection of the results. Automatic rank selection is still an open problem and needs to be explored through further research. TABLE I gives the spectral correlation and distribution correlation obtained from three algorithms for tumor and necrosis tissue types. From TABLE I, we can see for some datasets NMF and hNMF methods are unable to identify the tumor source, whereas NCPD methods succeeds in identifying tumor source and its distribution. In case of necrotic tissue the source correlation is almost identical for all three methods, whereas the NCPD has slightly better distribution correlation. Moreover, in case of tumor tissue, NCPD clearly has better source and distribution correlation compared to NMF and hNMF.

The result of one in-vivo example is shown in Fig. 3 . NCPD, NMF and hNMF methods are applied to a $16 \times 16$ voxel grid shown in second row of Fig. 3a. The spectra truncated to the region $0.25-4.2 \mathrm{ppm}$ are of length 517 . The reduced spectra are constructed using a window length $L=20$ and the window is moved by a stepsize of 5 samples. Therefore the size of the MRSI tensor $\mathcal{T}$ with $16 \times 16(256)$ voxels is $100 \times 100 \times 256$. The six sources and their corresponding distribution maps obtained using rank-6 NCPD and NMF methods are shown in Fig. 3b, 3c, 3d and 3e. With the hNMF method, only three sources are obtained, as shown in Fig. 3f and 3g. Fig. 3g shows that the hNMF method identifies the normal and necrotic tissue properly, but fails to recover the tumor tissue. Single-stage NMF identifies normal and necrotic tissue, but only the necrotic source is good and the normal source deviates a lot as shown in Fig. $3 d$ ( $1^{\text {st }}$ row).
TABLE I: First two columns: in vivo MRSI datasets and the relevant tissue type corresponding to the dataset. $\mathrm{T}$ and $\mathrm{N}$ represent tumor and necrosis, respectively. Columns 3 to 5 give the spectral correlations for NCPD, NMF and hNMF algorithms, respectively. Columns 6 to 8 give the distribution correlations. ' $\mathrm{X}$ ' denotes failure to recover corresponding tissue source. Last four rows show the median and median absolute deviation (MAD) over all datasets for tumor and necrosis, respectively.

\begin{tabular}{|c|c|c|c|c|c|c|c|}
\hline & \multicolumn{3}{|c|}{ Spectral correlation } & \multicolumn{3}{|c|}{ Distribution correlation } \\
\hline & & NCPD & NMF & hNMF & NCPD & NMF & hNMF \\
\hline \multirow{2}{*}{1} & $\mathrm{~T}$ & 0.9917 & 0.6592 & 0.7423 & 0.7743 & 0.7057 & 0.7015 \\
\hline & $\mathrm{N}$ & 0.9951 & 0.9900 & 0.9964 & 0.8965 & 0.8486 & 0.8585 \\
\hline \multirow{2}{*}{2} & $\mathrm{~T}$ & 0.9622 & $\overline{0.6110}$ & 0.9551 & 0.7879 & 0.6695 & 0.6908 \\
\hline & $\mathrm{N}$ & 0.9995 & 0.9974 & 0.9979 & 0.8947 & 0.8363 & 0.8779 \\
\hline \multirow{2}{*}{3} & $\bar{T}$ & $\overline{9911}$ & $\mathrm{X}$ & $\mathrm{X}$ & 0.8018 & $\mathrm{X}$ & $\bar{X}$ \\
\hline & $\mathrm{N}$ & 0.9975 & 0.9971 & 0.9972 & 0.9552 & 0.8949 & 0.8967 \\
\hline \multirow{2}{*}{4} & $\mathrm{~T}$ & 0.9918 & 0.5045 & 0.4481 & 0.8478 & 0.5117 & 0.5785 \\
\hline & $\mathrm{N}$ & 0.9963 & 0.9902 & 0.9962 & 0.8413 & 0.7389 & 0.7433 \\
\hline \multirow{2}{*}{5} & $\bar{T}$ & 0.9530 & $\bar{X}$ & $\bar{X}$ & 0.6152 & $\bar{X}$ & $\bar{X}$ \\
\hline & $\mathrm{N}$ & 0.9959 & 0.9937 & 0.9901 & 0.8538 & 0.4977 & 0.5949 \\
\hline \multirow{2}{*}{6} & $\bar{T}$ & 0.9809 & 0.6353 & 0.7618 & 0.7932 & 0.7990 & 0.7892 \\
\hline & $\mathrm{N}$ & 0.9985 & 0.9952 & 0.9970 & 0.8133 & 0.7162 & 0.7346 \\
\hline 7 & $\mathrm{~T}$ & 0.9352 & 0.7122 & 0.8515 & 0.7047 & 0.6115 & 0.5672 \\
\hline 8 & $\mathrm{~T}$ & 0.9756 & 0.8805 & 0.9429 & 0.8505 & 0.7813 & 0.6429 \\
\hline 9 & $\mathrm{~T}$ & 0.9795 & 0.8535 & 0.9748 & 0.8967 & 0.8670 & 0.7995 \\
\hline 10 & $\mathrm{~T}$ & 0.9895 & 0.6899 & 0.8923 & 0.7718 & 0.6658 & 0.6969 \\
\hline \multicolumn{2}{|c|}{ Median(T) } & 0.9802 & 0.6745 & 0.8719 & $\overline{0.7906}$ & 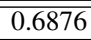 & 0.6938 \\
\hline \multicolumn{2}{|c|}{ MAD(T) } & 0.0112 & 0.0514 & 0.0930 & 0.0380 & 0.0849 & 0.0731 \\
\hline \multicolumn{2}{|c|}{ Median(N) } & 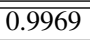 & $\overline{00.9945}$ & 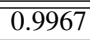 & $\overline{0.8742}$ & 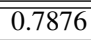 & $\overline{00.8009}$ \\
\hline \multicolumn{2}{|c|}{ MAD(N) } & 0.0013 & 0.0028 & 0.0005 & 0.0276 & 0.0662 & 0.0716 \\
\hline
\end{tabular}

NMF also fails to recover the tumor tissue. NCPD method identifies all three, tumor, necrotic and normal tissue types. Fig. $3 b$ and $3 c$ show that the estimated tissue sources and their corresponding spatial distributions are accurate when compared to expert labeling. In this example we estimate six sources and their corresponding distribution from rank6 NCPD. Three sources correspond to tumor, necrosis and normal tissue type, the other three sources correspond to artifacts (Fig. $3 \mathrm{~b}$ and $3 \mathrm{c}: 6^{\text {th }}$ row) and spectra from the outer edges of the voxel grid (Fig. $3 \mathrm{~b}$ and $3 \mathrm{c}: 3^{\text {rd }}$ and $4^{\text {th }}$ row), in other words, spectra of poor quality.

\section{Discussion AND CONCLUSION}

In this paper we have proposed a method to represent the 2D-MRSI data in a tensor using a reduced format of the spectra. A novel tissue type differentiation algorithm based on non-negative canonical polyadic decomposition with $l_{1}$ regularization was developed. To explore the clinical applicability of the proposed algorithm, it has been tested on 10 short-TE MRSI signals from patients having glioma. NCPD algorithm outperforms the existing tissue type differentiation methods based on NMF and hNMF. The advantage of this tensor method is that the construction of MRSI tensor couples the peaks in the spectra because of the $X X^{T}$ in the frontal slices. Therefore, in the spectral sources obtained from the NCPD algorithm the peaks will be coupled, i.e. we 

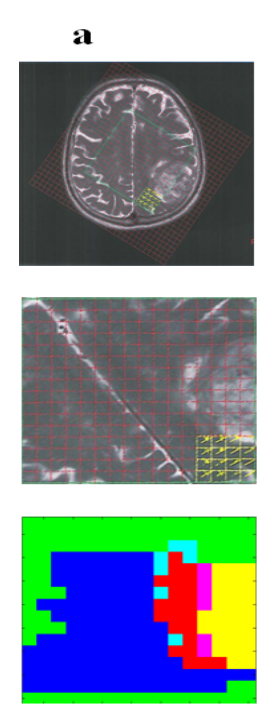

b
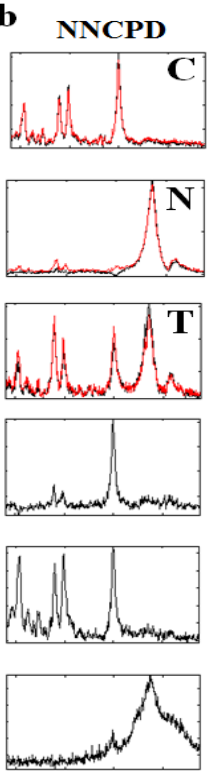

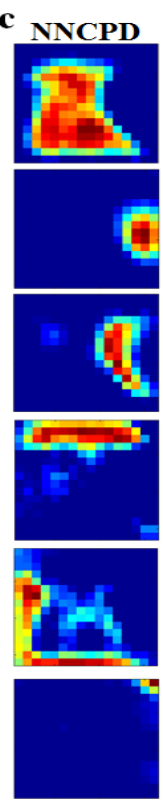

d
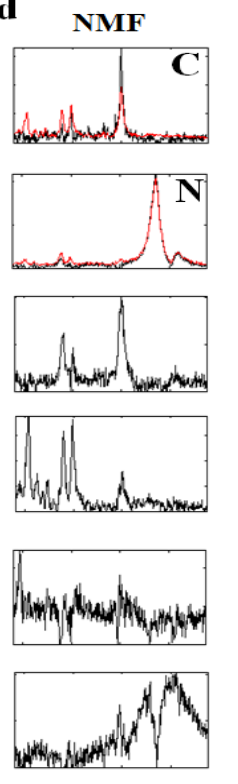

$\mathbf{f}$

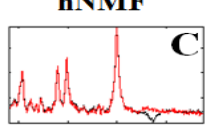

$\int_{h}^{\mathbf{N}}$
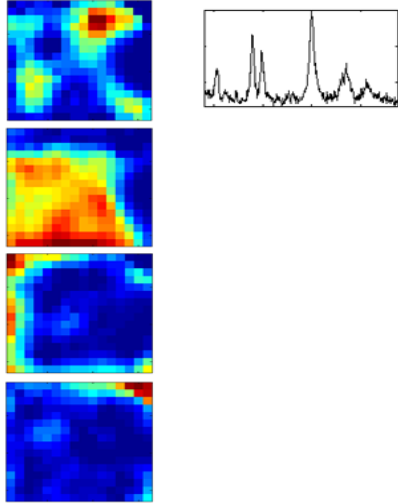

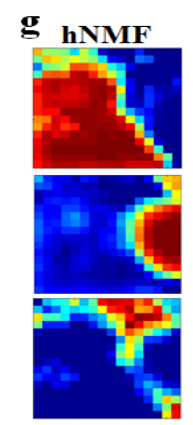

Fig. 3: Tissue pattern differentiation using ${ }^{1} \mathrm{H}$ MRSI: $\mathrm{C}$, $\mathrm{T}$ and $\mathrm{N}$ represent normal, tumor and necrosis, respectively. (a) First row: T2-weighted anatomical MR image of a brain tumor with areas of necrosis. Second row: voxels within the MRSI excitation volume superimposed on anatomical image. Third row: expert labeling, where yellow indicates $\mathrm{N}$, red indicates $\mathrm{T}$, magenta indicates $\mathrm{T} / \mathrm{N}$, dark blue indicates $\mathrm{C}$, light blue indicates $\mathrm{C} / \mathrm{T}$ and green indicates spectra of poor quality. (b, c) results of NCPD. (b) The recovered sources from NCPD method are shown in black. First three rows represent C, N and $\mathrm{T}$ spectral sources in black, with tissue specific spectra based on expert labeling overlaid in red. The remaining three rows represent artifacts and spectra from outer edges. (c) Distribution maps corresponding to spectral profiles in (b). (d, e) Results of single-stage NMF. (d) The recovered sources are shown in black, overlaid with the expert-based tissue specific spectra in red. First two rows show control and necrosis spectra and the remaining rows show other spectra obtained using rank-6 NMF. (e) Distribution maps corresponding to (d). (f, g) Results of hNMF. (f) Recovered sources shown in black and expert-based tissue specific spectra in red. First two rows show control and tumor spectra. (g) Distribution maps corresponding to (f).

will not get individual peaks as sources. The performance of the hNMF is not so good because the voxels in the outer edge of MRSI excitation volume are included in the assessment, whereas NCPD can account for artifacts and bad voxels present in the outer edges because more sources $(R=6)$ are used in the decomposition. NCPD algorithm has the potential to replace the hNMF method in unsupervised nosologic imaging for brain tumors [12], which can be used as a tool to assist brain tumor diagnosis.

\section{ACKNOWLEDGMENT}

Authors would like to thank radiologist Dr. Sofie Van Cauter for labeling the MRSI voxels.

\section{REFERENCES}

[1] Y. Li, D. M. Sima, S. V. Cauter, C. Sava, R. Anca, U. Himmelreich, Y. Pi, and S. Van Huffel, "Hierarchical non-negative matrix factorization (hNMF): a tissue pattern differentiation method for glioblastoma multiforme diagnosis using MRSI," NMR in Biomedicine, vol. 26, no. 3, pp. 307-319, 2013.

[2] S. Dimou, R. Battisti, D. Hermens, and J. Lagopoulos, "A systematic review of functional magnetic resonance imaging and diffusion tensor imaging modalities used in presurgical planning of brain tumour resection," Neurosurgical review, vol. 36, no. 2, pp. 205-214, 2013.

[3] F. S. De Edelenyi, A. Simonetti, G. Postma, R. Huo, and L. Buydens, "Application of independent component analysis to $1 \mathrm{H}$ MR spectroscopic imaging exams of brain tumours," Analytica Chimica Acta, vol. 544, no. 1, pp. 36-46, 2005.
[4] T. Laudadio, P. Pels, L. De Lathauwer, P. Van Hecke, and S. Van Huffel, "Tissue segmentation and classification of MRSI data using canonical correlation analysis," Magnetic Resonance in Medicine, vol. 54, no. 6, pp. 1519-1529, 2005.

[5] T. Laudadio, N. Mastronardi, L. Vanhamme, P. Van Hecke, and S. Van Huffel, "Improved Lanczos algorithms for blackbox MRS data quantitation," Journal of Magnetic Resonance, vol. 157, no. 2, pp. 292-297, 2002.

[6] J. B. Poullet, "Quantification and classification of magnetic resonance spectroscopic data for brain tumor diagnosis," Ph.D. dissertation, $\mathrm{PhD}$ Thesis. Leuven, Belgium, 2008.

[7] V. Govindaraju, K. Young, and A. A. Maudsley, "Proton NMR chemical shifts and coupling constants for brain metabolites," $N M R$ in Biomedicine, vol. 13, no. 3, pp. 129-153, 2000.

[8] A. Cichocki, R. Zdunek, A. H. Phan, and S. i. Amari, Nonnegative matrix and tensor factorizations: applications to exploratory multiway data analysis and blind source separation. John Wiley \& Sons, 2009.

[9] L. Sorber, V. Barel, and L. D. Lathauwer, "Tensorlab v2.0," Available online, January 2014.

[10] L. Sorber, M. Van Barel, and L. De Lathauwer, "Structured data fusion," Selected Topics in Signal Processing, IEEE Journal of, vol. 9, no. 4, pp. 586-600, June 2015.

[11] K. Koh, S. Kim, and S. Boyd, "11_ls: A matlab solver for large-scale L1-Regularized least squares problems," http://web.stanford.edu/ boyd/11_ls/, 2008.

[12] Y. Li, D. M. Sima, S. Van Cauter, U. Himmelreich, A. R. C. Sava, Y. Pi, Y. Liu, and S. Van Huffel, "Unsupervised nosologic imaging for glioma diagnosis," Biomedical Engineering, IEEE Transactions on, vol. 60, no. 6, pp. 1760-1763, 2013. 